\title{
Malignant cardiac neoplasms are rare but lethal
}

\author{
Jaouadi Abdelaziz ${ }^{1}$, Ahmad Jamal ${ }^{1}$, Marwaa Abdallah ${ }^{1}$, Messaoudi Yosra $^{1}$, Bilel \\ Ibnezzine $^{1}$, and Nejeh Benhalima ${ }^{1}$ \\ ${ }^{1}$ Ibn Jazzar University Hospital
}

April 27, 2020

\begin{abstract}
Background: Primary cardiac neoplasms are rare and occur less commonly than metastatic disease of the heart which sarcomas are the largest group of them and have a mesenchymal origin. metastases to or direct invasion of the heart are far more common, and many tumor types are reported in the literature such as breast cancer, lung cancer, melanoma and various sarcomas. The prognosis after surgery is usually excellent in the case of benign tumors, but the prognosis of malignant tumors remains dismal. Patients and methods: We are about to report through 5 cases of malignant cardiac tumors hospitalized in our department and treated later with cardiac surgery, the subtypes of cardiac masses and their diagnosis and prognosis (follow-up) features with literature review. Results: Five cases of cardiac neoplasms had been reported in this work; that can be classified in one case of primary cardiac origin and 4 cases as secondary to metastases (breast cancer, lung cancer, osteosarcoma and mediastinal thymoma). Echocardiogram was the main exploration technique to be performed and thoracic CT was performed in all cases. Cardiac surgery was the main treatment in only one case, but the others palliative treatment was the case. Conclusion: Malignant cardiac masses are infrequent and often asymptomatic, most of the time they are secondary to extra cardiac tumor. Early diagnosis of cardiac tumors necessitates a high level of suspicion. Therefore, surgery remains the cornerstone in the therapy of cardiac sarcomas and it should be attempted once it is technically feasible.
\end{abstract}

\section{Background:}

Primary cardiac neoplasms are rare and occur less commonly than metastatic disease of the heart which sarcomas are the largest group of them and have a mesenchymal origin. metastases to or direct invasion of the heart are far more common, and many tumor types are reported in the literature such as breast cancer, lung cancer, melanoma and various sarcomas. The prognosis after surgery is usually excellent in the case of benign tumors, but the prognosis of malignant tumors remains dismal.

\section{Patients and methods:}

CASE 1:

39 years old female without history of cardiovascular diseases, who was admitted with recent onset of shortness of breath, on examination BP was $110 \backslash 80 \mathrm{~mm} \mathrm{hg}$, regular heart rate at 118 beats $\backslash$ min, there was no abnormal findings on pulmonary auscultation, but heart beats were not audible and there were signs of right sided heart failure. Chest-x-ray didn't show any special anomalies and electrocardiogram was normal except for sinus tachycardia at 120 beats $\backslash$ min.

In the echocardiography there was a mass with an heterogenous texture multilobulated that measure $6.5 \mathrm{~cm}$ in his high axis which occupies the right atrium and diving in the right ventricle to block the tricuspid valve. There was also a massive pericardial effusion with tamponade findings. (fig1-2)

A CT thoracic scan was performed for further diagnosis has confirmed the findings of the echocardiography which was a mass located in the right atrium extended to the inferior vena cava but did not occlude it (fig 3) 
Patient was transferred to cardiac surgery department the same day where she was clinically stabilized and pericardiocentesis was performed to remove $1200 \mathrm{cc}$ of liquid. The next day she was operated and at the time of surgery, the right atrial appendage was noted to be very congested and the right atrial cavity was found to be replaced by a friable tumor which had "fronds like" appearance more over right ventricle "s free wall was infiltrated but tricuspid valve was free of the tumor. Then, the entire free wall of right atrium and the tumor were excised extending from and to the origin of vena cava. A sample of the tumor was subjected to frozen section examination which suggested the diagnosis of sarcoma. Then, the right atrium was reconstructed using autologous pericardium. (fig 4-5)

Subsequently; Macro and microscopic study has revealed the diagnosis of mild differentiated primitive cardiac angiosarcoma.

After recovery from surgery the patient was submitted to chemotherapy and tumor surveillance plan involving serial echocardiograms is planned.

\section{CASE 2:}

70 years old male with one-year history of right arm amputation who comes to the emergency department for a rapidly progressive worsening dyspnea. On examination, the patient's BP was at $70 / 40 \mathrm{~mm}$ hg with peripheral signs of shock and irregular tachycardia at 140 BPM. There were signs of acute right sided heart failure and polypnea at $28 \mathrm{CPM}$.

Electrocardiogram showed tachyarrhythmia at 140 BPM with aberrancy. The cardiothoracic index at the chest $\mathrm{X}$ ray was at 0.6 without signs for pulmonary edema.

Biologically there was hypoxemia and respiratory alkalemia on the arterial blood gases.

Pulmonary embolism was highly suggested, so an echocardiography was performed and showed an enormous irregular and heterogenous mass $\left(13^{*} 6 \mathrm{~cm}\right)$ which occupied all the right cavity from the right atrium to the right ventricle through the tricuspid valve. (fig 6-7)

Questioning the family revealed the diagnosis of an osteosarcoma of the right humerus infiltrating neighboring soft tissues which was amputated without any secondary metastatic sites.

Thoracic CT was performed to confirm the extension of this mass to cardiac cavities and the two main vena cava which they were occluded by the tumor. There was also metastatic lesion in the right liver part.

The diagnosis of a metachronous metastatic osteosarcoma to the right heart complicated mimicking an acute pulmonary embolism was tagged.

The patient refused any medical or mini invasive treatment and died few days later.

CASE 3:

50 years old male without medical history consulted the emergency department for shortness of breath. On examination, cardiac frequency was at $110 \mathrm{BPM}$, BP was at 120/80 $\mathrm{mm}$ hg. no abnormal cardiac murmur on auscultation but there was a muffling of heart sounds.

A close examination of the patient had revealed a superior vena cava syndrome (blockpnea, superficial venous circulation and cyanosis).

Electrocardiogram didn't show any special abnormalities, but an echocardiogram was performed and showed a mild to important pericardial effusion.

The patient has been hospitalized and non-steroid and steroid anti-inflammatory treatments was putted. The next day there were worsening of the dyspnea so another TTE has revealed an abnormal mass in the pericardial cavity.

CT thoracic angiography was performed the same day has showed an anterior mediastinal mass with calcified and necrotic sites extended to the pericardium and to the superior vena cava. (fig 8) 
Scanner guided transthoracic biopsy was performed from this mass to confirm the diagnosis of thymoma and the patient was putted under chemotherapy. Two pericardiocentesis are attempted in the curse for a massive pericardial effusion then a pleural-pericardial window was done to minimalize the mass effect.

Serial echocardiography was programmed for the follow up.

\section{CASE 4:}

43 years old female diagnosed for one year with breast cancer treated with Patey intervention. Palliative treatment was initiated since the last 2 months when the diagnosis of tumor recurrence was established. In the past 1 week, she suffers from dyspnea had been worsened progressively.

Currently, she consulted the emergency department for acute dyspnea, on examination her BP was at 80/40 $\mathrm{mm}$ hg with signs of hypovolemic shock and tachycardia at $130 \mathrm{bpm}$.

Echocardiography found a massive pericardial effusion with compression signs on right cavities. Tamponade was the clear diagnosis, so an urgent pericardiocentesis was performed and $1400 \mathrm{ml}$ of liquid was retrieved from the pericardial cavity. In the aftermath, there was a significant improvement of her hemodynamic and respiratory states.

Later, patient was hospitalized in our department to survey her hemodynamical parameters and initiates palliative chemotherapy.

Full body CT was performed had discovered multi-sites metastatic cancer (bones, lungs, liver...) with regional extension to the pericardium.

In the following 4 days, recurrence of the pericardial effusion was massive and 2 more pericardiocentesis followed with continuous drainout were performed but recurrences of this massive effusion had motivated us to make pleuro-pericardial window.

After two weeks she died with a respiratory distress after the occlusion of the pleuro-pericardial window with the neoplasm tissue.

\section{CASE 5:}

45 years old male, heavy smoker, consulted the emergency department for sub-acute dyspnea and syncope, on examination his BP was at 100/70, tachycardia at $100 \mathrm{bpm}$ and polypnea at $25 \mathrm{cpm}$, without peripheral signs of shock. There were also some rales at pulmonary auscultation.

Electrocardiogram didn't show any anomalies except for sinus tachycardia at $110 \mathrm{bpm}$. Chest $\mathrm{x}$ ray showed bilateral pulmonary edema, some apical opacities, bilateral pleural effusion and mediastinal enlargement.

TTE was performed on the field showed a heterogenous mass occupied the left atrium and diving into the left ventricle to hinder the cardiac output. Right cavities were dilated with high systolic pulmonary pressure and moderate pericardial effusion.

Thoracic CT showed large pulmonary opacity suggest a lung cancer that had infiltrated the mediastinum then the pericardium and cardiac cavities

The patient was then hospitalized in our department after refusing the cardiac surgery but in the following day he had a refractor cardiogenic shock and died.

\section{RESULTS:}

Five cases were discussed in this work; through them we report their clinical, diagnosis and prognosis features.

In all cases their clinical presentation was about dyspnea associated to cardiogenic shock (CS) in two cases and without CS in the other cases. Signs of right sided heart failure were present in two case despite signs of left sided heart failure were present in only one case. 
Echocardiogram was the main exploration technique to be performed; right sided masses were present in two cases; one mass was in left cavities; the other cases were presented as moderate to massive pericardial effusion, but echocardiographic findings of tamponade were diagnosed in two cases.

Thoracic CT was performed in all cases for further diagnosis and to know origin and extension of the tumor.

There was one case of primary cardiac tumor as angiosarcoma, the others were secondary metastatic tumors to the heart cavities (osteosarcoma, mediastinal thymoma, breast cancer and lung cancer).

Two patients were survived, and they are under chemotherapy (after cardiac surgery in the angiosarcoma case). The others were died in the following days after the hospitalization.

\section{Discussion:}

The first heart tumors were described by Colomnus in the 15th century and by Boneti in the 18th century. Alberts documented for the first time in 1835 resection of a cardiac tumor.

Primary tumors of the heart, except for atrial myxomas, are very rare (a prevalence of 0.001-0.28\%) [1]; metastases to or direct invasion of the heart are far more common.

Lam KY et al has published in 1993 a review of 12,485 autopsies and has revealed only 7 cases of primary heart neoplasms, whereas the investigators found 154 cases of metastatic heart tumors [2].

The common family of primary cardiac neoplasms are soft tissue sarcomas. Angiosarcomas are the most frequent type then we have rhabdomyosarcomas, malignant mesotheliomas and fibrosarcoma. Other types are rarer like leiomyosarcomas, liposarcomas and osteosarcomas $[1,3,4]$.

Secondary neoplasms of the heart are 20 to 40 times more common than primary cardiac malignancies [5].

According to Burke and Virmani, among their data of 6,240 autopsies, 953 were identified as cardiac metastases [6], since the absolute number of cases of each original malignancy was different, lung cancer, breast cancer, lymphoma, leukemia, and esophageal cancer had a high number of metastatic cases. while high frequency of metastasis was seen in malignant melanoma, malignant germ cell tumors, and leukemia [6].

The most common clinical presentation is heart failure, followed by symptoms caused by peripheral emboli to the cerebral, systemic and coronary arterial circulatory systems [10].

Echocardiography is the best diagnostic tool for intracavity and mural cardiac tumors. It can identify involvement of the valves and their competency, ventricular function, irregular pericardial thickening and intracavity masses interfering with blood flow. It also can evaluate the size and the extension of cardiac neoplasms $[6,7,8]$.

Computed tomography (CT), magnetic resonance imaging (MRI), and/ or angiography further adds to the diagnostic information especially for intrapericardial tumors.

Cardiac catheterization is recommended in all patients over 40 years of age presenting with intracardiac masses and in all patients with large right atrial masses [12].

Malignant tumors of the heart usually require a cardiac surgery for debulking the tumor as either for palliative treatment or for tissue diagnosis. They are often requiring palliative surgery, because $78 \%$ of the patients present with a mechanical blood flow obstruction $[9,10,11]$.

If complete resection is possible, surgery followed by adjuvant chemotherapy provides better palliation and can improve survival [13].

\section{Conclusion:}

Malignant cardiac masses are infrequent and often asymptomatic, most of the time they are secondary to extra cardiac tumor. Early diagnosis of cardiac tumors necessitates a high level of suspicion. Therefore, 
surgery remains the cornerstone in the therapy of cardiac sarcomas and it should be attempted once it is technically feasible.

Keywords: Sarcomas, metastasis, surgery, chemotherapy, echocardiography

Figures legends:

Fig 1-2: Short axis and apical TTE views shows a mass in the right heart with an important pericardial effusion.

Fig 3: CT scan view that shows the tumor infiltrating the right atrium and the right ventricle

Fig 4-5: macroscopic picture of the mass extracted from the right cavities.

Fig 6-7: four cavities chamber view and short axis view shows an important mass in the right cavities.

Fig 8: A CT scan shows a mass in the anterior mediastinum

\section{References}

1. McAllister HA, Fenoglio JJ. Tumors of the cardiovascular system. In: Atlas of tumor pathology, second series. Washington: Armed Forces Institute of Pathology; 1978.

2. Lam KY, Dickens P, Chan AC. Tumors of the heart. A 20-year experience with a review of 12,485 consecutive autopsies. Arch Pathol Lab Med 1993; 117:1027-1031.

3. Vander Salm TJ. Unusual primary tumors of the heart. Semin Thorac Cardiovasc Surg 2000; 12:89e100.

4. Laissy JP, Fernandez P, Mousseaux E, Dacher JN, Crochet D Cardiac tumors. J Radiol 2004; 85:363e369.

5. Hallahan DE, Vogelzang NJ, Borow KM, Bostwick DG, Simon MA. Cardiac metastases from soft-tissue sarcomas. J Clin Oncol 1986; 4:1662-1669.

6. Burke A, Virmani R. Classification and incidence of cardiac tumors. Tumors of the heart and great vessels. In: Burke A, Virmani R, editors. Atlas of tumor pathology, series 3, fascicle 16. Washington, D.C.: Armed Forces Institute of Pathology; 1996. pp. 195-209.

7. Putnam JB Jr, Sweeney MS, Colon R, Lanza LA, Frazier OH, Cooley DA. Primary cardiac sarcomas. Ann Thorac Surg 1991;51: 906-910.

8. Fine G. Neoplasms of the pericardium and heart. In: Gould SE, editor. Pathology of the heart and blood vessels. Springfield, IL: Charles C. Thomas; 1968. p. 851.

9. Grebenc ML, Rosado de Christenson ML, Burke AP, Green CE, Galvin JR. Primary cardiac and pericardial neoplasms: radiologic-pathologic correlation. Radiographics 2000; 20: 1073-103.

10. Ekmektzoglou KA, Sameli GF, Xanthos T. Heart and tumors: location, metastasis, clinical manifestations, diagnostic approaches and therapeutic considerations. J Cardiovasc Med. 2008; 9:769-77.

11. Miralles A, Bracamonte L, Soncul H, Diaz del Castillo R, Akhtar R, Bors V, et al. Cardiac tumors: clinical experience and surgical results in 74 patients. Ann Thorac Surg. 1991; 52: 886-95.

12. Butany J, Nair V, Naseemuddin A, Nair GM, Catton C, Yau T. Cardiac tumours: diagnosis and management. Lancet Oncol 2005; 6: 219-228

13. Putnam JB Jr, Sweeney MS, Colon R, Lanza LA, Frazier OH, Cooley DA. Primary cardiac sarcomas. Ann Thorac Surg 1991;51: 906-910. 

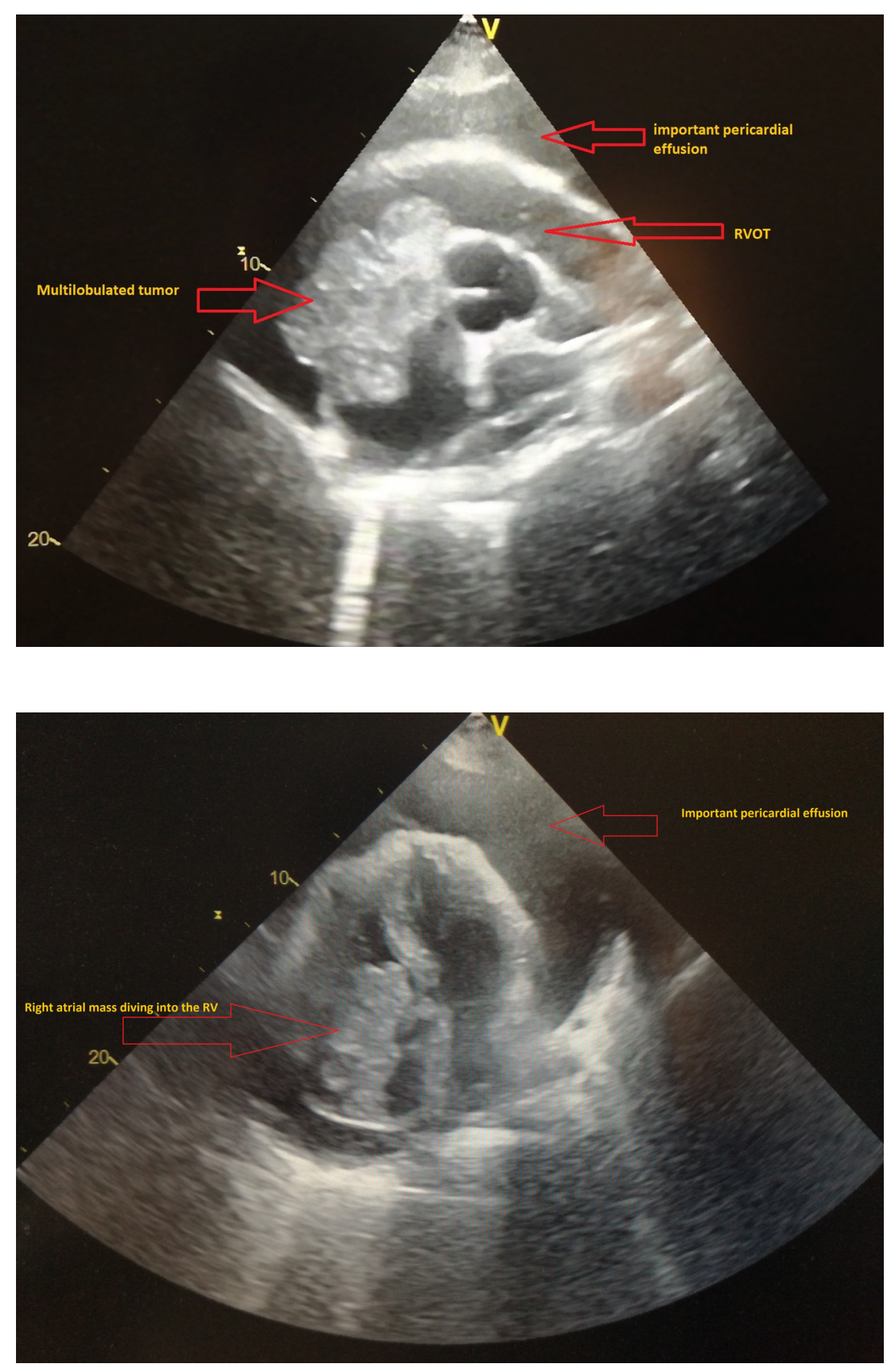


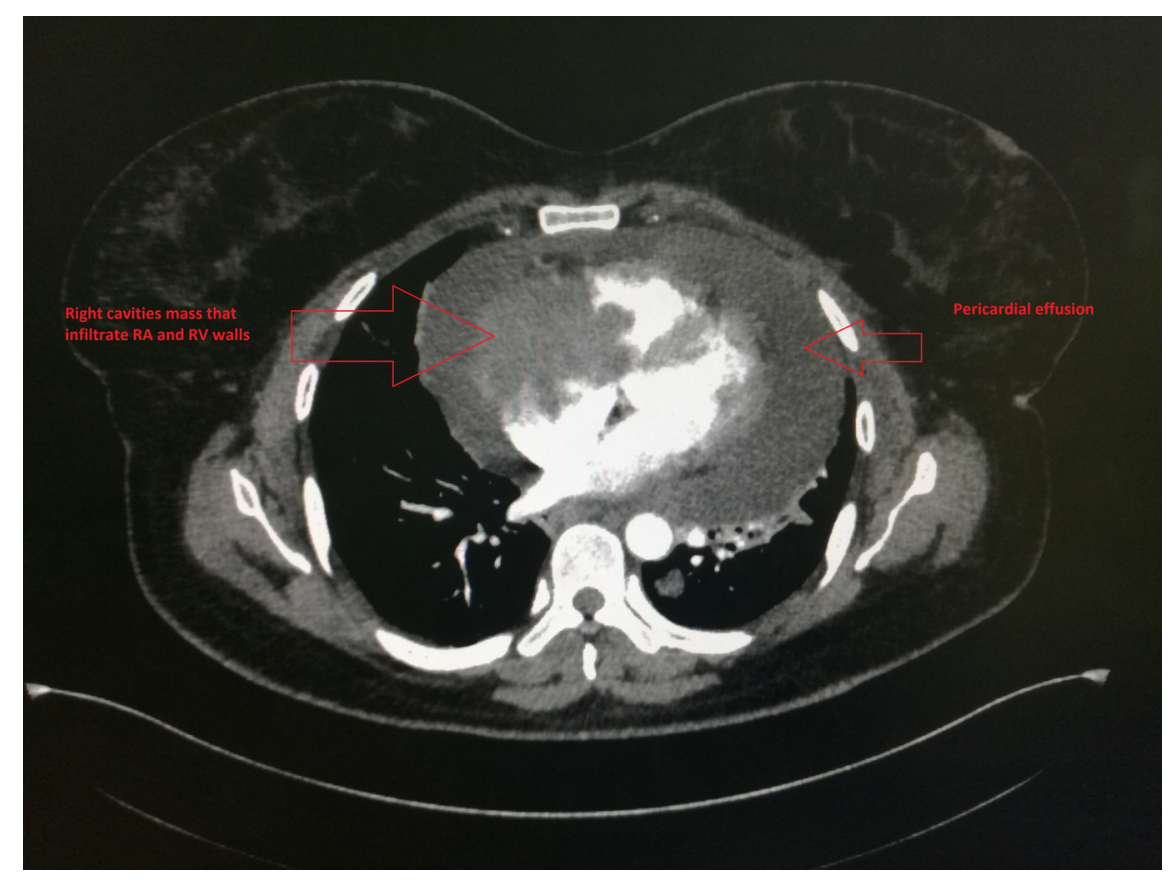




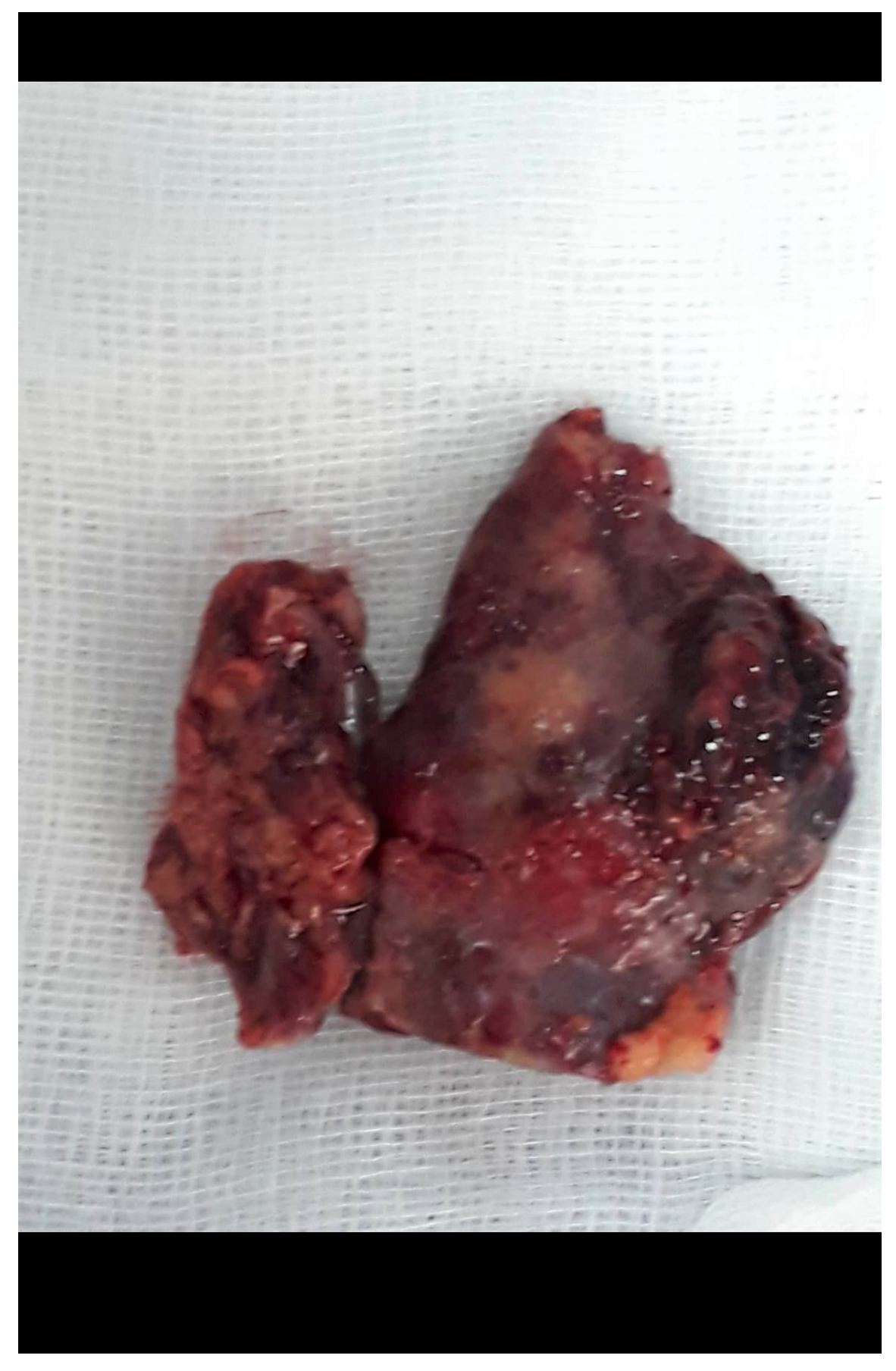




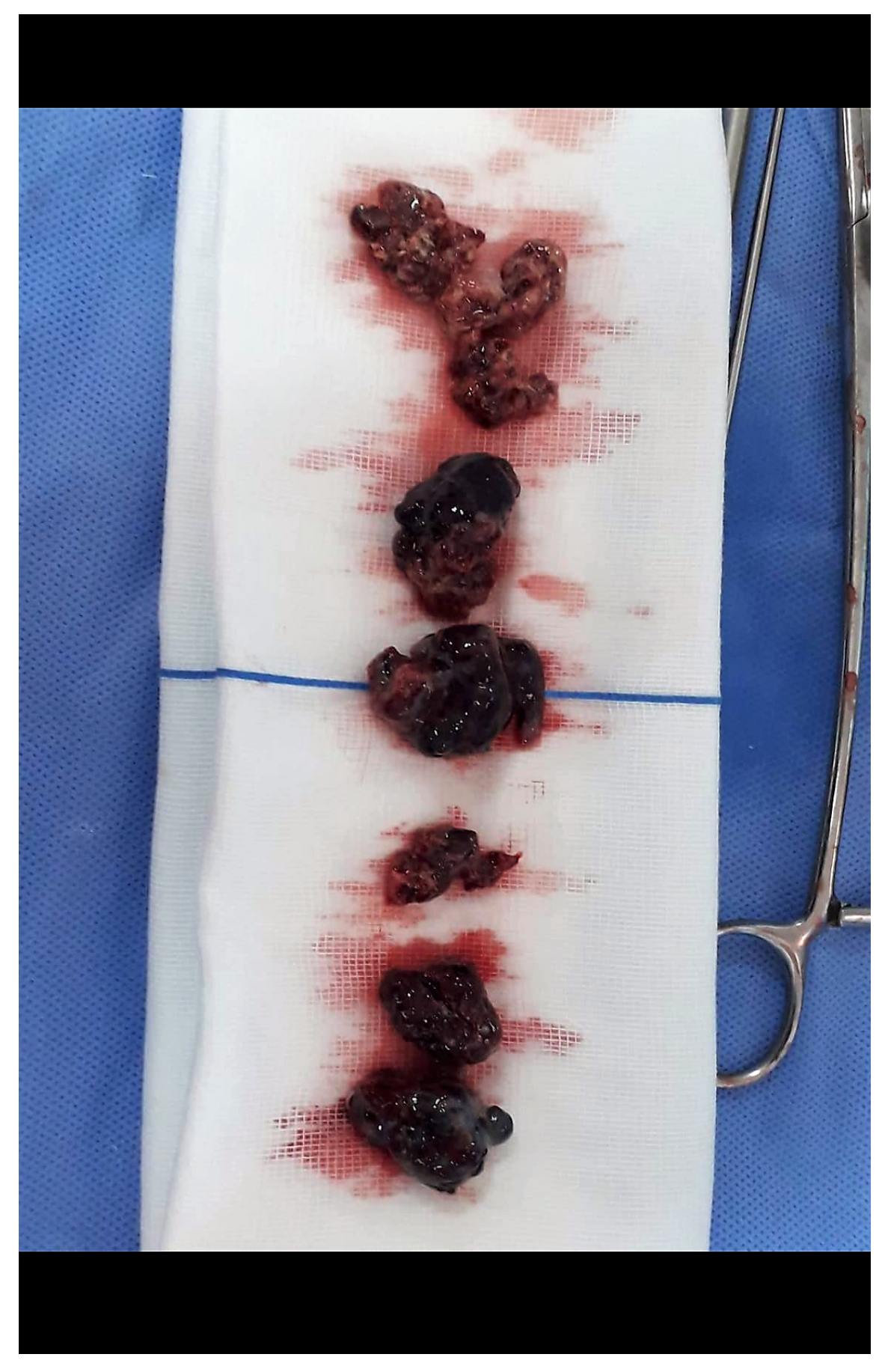



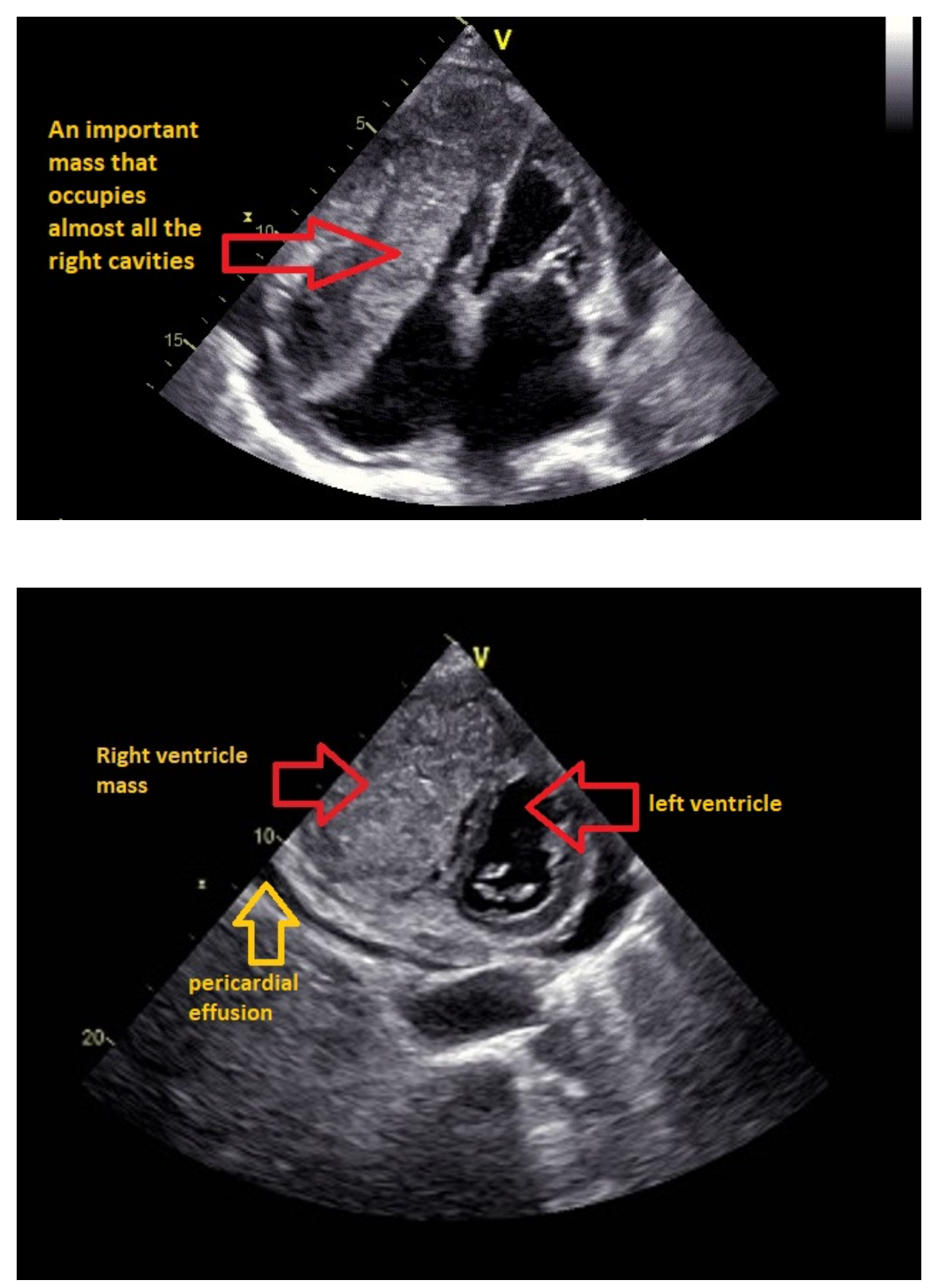


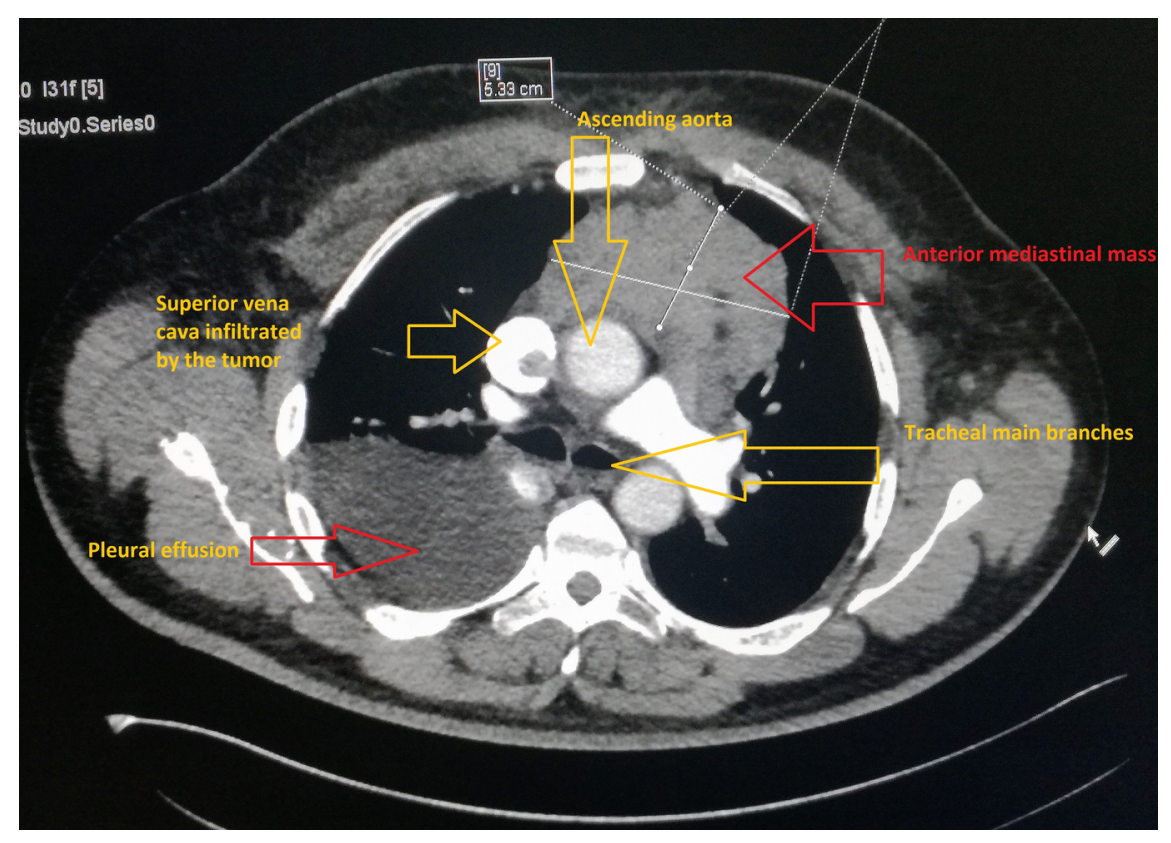

